\title{
Tests electrofisiológicos en el estudio de la patología visual
}

\section{Electrophysiological tests in the assessment of visual disorders}

\author{
M.I. Iragui-Madoz
}

\section{RESUMEN}

Los tests electrofisiológicos ayudan al estudio de la función visual. El objetivo de este trabajo es presentar las bases fisiológicas, aspectos técnicos e indicaciones generales de los tests que se practican de forma habitual en nuestro medio.

El electorretinograma-flash refleja la función de los fotorreceptores y de capas más externas de la retina. El electrorretinograma-patrón informa de las células ganglionares retinianas y puede identificar alteraciones maculares que simulan neuropatías ópticas. El electrooculograma valora la función del epitelio pigmentario y capas externas de la retina y diferencia la enfermedad de Best de otras bestrofinopatías. Los potenciales evocados visuales informan de las vías ópticas y son, sobre todo, útiles en la valoración del nervio óptico.

Palabras clave. Electrorretinograma-flash. Electrorretinograma-patrón. Electrooculograma. Potenciales evocados visuals.

\begin{abstract}
Some electrophysiological tests are helpful in the assessment of visual disorders. The physiological basis, technical aspects and general indications of those available at most neurophysiology services are reviewed.

The Full-Field electroretinogram reflects photoreceptor and outer retinal function. The Pattern electroretinogram assesses retinal ganglion cell function and can identify macular dysfunction mimicking optic nerve disease. The electro-oculogram is a test of function of the outer retina and retinal pigment epithelium; it differentiates Best's disease from all other bestrophinopathies. Visual evoked potentials evaluate the visual pathway and are especially helpful in the evaluation of the optic nerve.
\end{abstract}

Key words. Full-field electrretinogram. Pattern electroretinogram. Electrooculogram. Visual evoked responses.
Servicio de Neurofisiología Clínica. Hospital Virgen del Camino. Pamplona.

\section{Correspondencia}

María Ignacia Iragui-Madoz

Servicio de Neurofisiología Clínica

Hospital Virgen del Camino

Irunlarrea, 4

31008 Pamplona

Tfno. 848429620

E-mail: miraguim@cfnavarra.es 


\section{INTRODUCCIÓN}

Los tests electrofisiológicos son técnicas complementarias útiles para detectar y localizar alteraciones de la retina y de las vías visuales. Han sido ampliamente estudiados, pero aquí no se pretende hacer una revisión exhaustiva de los mismos ni de las diversas patologías en las que están indicados, sino exponer sencillamente las indicaciones generales y su fundamento.

\section{NOCIONES ANATOMOFISIOLÓGICAS}

La percepción visual se inicia en la retina. La luz que penetra a través de la córnea se proyecta en la parte posterior del ojo, en la retina, y ésta la convierte en señales eléctricas que son enviadas, a través del nervio óptico, a los centros superiores del cerebro donde se concluye el procesamiento necesario para la percepción ${ }^{1}$.

La retina es una parte del sistema nervioso central porque deriva de la placa neural del ectodermo. Los primeros signos del desarrollo del ojo aparecen en la tercera semana de gestación con un esbozo de las vesículas ópticas que posteriormente crecen y se invaginan. La pared externa de la vesícula formará el epitelio pigmentario, que a las ocho semanas está completo y pigmentado La pared interna dará lugar a la retina y a los elementos gliales. Del margen nacerán los elementos neuro-epiteliales del iris, cuerpo ciliar y borde de la pupila. El desarrollo de los diferentes estratos y tipos celulares se va produciendo desde la pared interna que, a su vez, se diferencia en dos capas: una interna y otra externa. La interna dará las células ganglionares con sus axones, las de Müller, las amacrinas y, más tarde, las bipolares y horizontales; de la externa se formarán los fotorreceptores. El desarrollo se produce desde el centro hacia la periferia y no se completa hasta los 5-6 años de vida postnatal ${ }^{2}$.

Los fotorreceptores forman la capa más externa de la retina, por delante del epitelio pigmentario, y como la luz tiene que atravesar todo el espesor de la retina para llegar a ellos, los axones de las capas proximales son amielínicos, relativamente transparentes. Hay dos tipos de fotorreceptores, conos y bastones. Los bastones, en relación a los conos, son más sensibles y capturan más luz, son capaces de detectar fotones únicos, son más sensibles a la luz dispersa, contienen un tipo de fotopigmento y las respuestas son más lentas. Los conos son más sensibles a la luz axial directa, necesitan más luz, producen respuestas rápidas y se distinguen tres tipos, S, M, y L, cada uno con un fotopigmento distinto, más sensible a una región concreta del espectro de luz visible (longitud de onda corta, media y larga -azul, verde y rojo- respectivamente). Su distribución no es homogénea: los bastones predominan en la retina periférica; sin embargo, en la zona más central, la fóvea, hay gran densidad de conos, si bien el $90 \%$ de los conos son extrafoveales y en conjunto mucho menos numerosos que los bastones. Los bastones son responsables de la visión nocturna; los conos de la diurna y de la visión cromática ${ }^{1-3}$.

La luz activa el fotopigmento e inicia una serie de fenómenos bioquímicos complejos que llevan a la hiperpolarización de los fotorreceptores (conos y bastones), disminuyendo la cantidad del transmisor químico que éstos emiten a las células bipolares. La célula bipolar de tipo "on" se despolariza y aumenta la cantidad de trasmisor químico que emite a la célula ganglionar "on". Ocurre lo contrario con la célula bipolar "off». Las células horizontales se localizan a nivel de las sinapsis fotorreceptor-célula bipolar (estrato plexiforme externo) y las amacrinas se localizan a nivel de las sinapsis célula bipolar-célula ganglionar (estrato plexiforme interno) y ambas realizan funciones de conexión lateral y modulación. Así, las señales de los fotorreceptores llegan hasta las células ganglionares El número de sinapsis que recibe una célula ganglionar es menor cuanto más central es su localización: la activación de uno o pocos conos foveales convergen en una célula ganglionar; en cambio, una célula ganglionar periférica recibe la información de muchísimos fotorreceptores. Por lo tanto, el campo receptivo -área de la retina que influye en una célula ganglionar- es menor 
cuanto más central es su localización. Las células ganglionares producen potenciales de acción y son de dos tipos: «de centro conectado" y "de centro desconectado". Las de centro conectado se excitan y aumenta su frecuencia de descarga cuando la luz llega al centro de su campo receptivo y se inhiben y desciende su frecuencia de descarga cuando la luz llega a la periferia de su campo receptivo. Las de centro desconectado, también llamadas conectadas por la periferia, tienen el comportamiento inverso. Esta organización contribuye a detectar cambios rápidos de iluminación y pequeños de contraste.

Los axones de las células ganglionares forman el nervio óptico (NO). Después de que los axones provenientes de las hemirretinas nasales se hayan cruzado en el quiasma, la información es llevada, en su mayor parte, al núcleo geniculado lateral del tálamo (NGL), a los estratos magno (M) y parvocelulares (P). Las células P son esenciales para la visión en color y la que requiere una resolución espacial rápida; las M para la que requiere resolución temporal rápida. Otra parte es llevada al tubérculo cuadrigémino superior, que controla las sacudidas oculares y al pretecho mesencefálico para control de los reflejos pupilares.

Del NGL las vías M y P llegan al lóbulo occipital, a la corteza visual primaria que está organizada en módulos funcionales. La corteza elabora la información por separado y descompone cada parte del campo visual en segmentos lineales de diferente orientación, procesando el color y combinando las aferencias de los dos ojos. Luego, por una vía dorsal, la mayoría de aferencias M llegan a la corteza parietal posterior, quedando relacionada con el movimiento y la profundidad; el resto de las aferencias $\mathrm{M}$ y las aferencias $\mathrm{P}$, por una vía ventral, llegan a la corteza temporal inferior que analizará datos de forma y color ${ }^{1,2,4-6}$.

\section{ELECTRORRETINOGRAMA}

El electrorretinograma (ERG) es el registro de la respuesta eléctrica de la retina a estímulos luminosos. Según el tipo de estímulo puede realizarse ERG-flash, también llamado "de estimulación difusa" o "de campo completo", ERG mediante patrón geométrico tipo damero y ERG multifocal

\section{ELECTRORRETINOGRAMA- FLASH (ERG-F)}

Los primeros datos se remontan a Holmgren, que en 1865 lo obtuvo en ranas, pero su uso clínico se difunde a partir de 1941 cuando Riggs diseña una lente de contacto y Karpe, en 1945, publica una serie de registros en ojos sanos y patológicos. Posteriormente Berson realza la importancia de medir tiempos implícitos además de las amplitudes de las respuestas y Gouras la de separar las respuestas de conos y de bastones ${ }^{7}$.

Pero las respuestas varían mucho dependiendo del estado previo de la retina, de las características del estímulo utilizado y del acceso de la luz, lo que hace muy difícil la comparación entre unos registros y otros. Con el fin de reducir todo lo posible estas variables, en 1989 se redactó un protocolo estándar que posteriormente la Sociedad Internacional de Electrofisiología Clínica de la Visión (ISCEV) ha ido revisando y concretando, siendo la revisión más reciente la de $2008^{8}$. Es un protocolo de mínimos para todas las exploraciones, dejando abierta la posibilidad de ampliarlo si se cree oportuno.

Aunque hay estudios realizados con electrodos de superficie y sin dilatación pupilar $^{9}$, el protocolo ISCEV exige dilatación para estimular toda la retina $\mathrm{y}$, preferentemente, electrodo de lentilla corneal desechable con referencia al canto externo del ojo. Se realiza adaptación previa a la oscuridad y se estimula mediante una campana "ganzfeld" con un flash de 0,01 cd.s.m ${ }^{-2}$, capaz de estimular los bastones, pero no suficiente para estimular conos. Se obtiene una onda "b», positiva. A continuación se estimula con 3 cd.s.m ${ }^{-2}$, que activa bastones y conos obteniendo una onda "a" negativa, seguida de la "b" positiva de mucha mayor amplitud y una "c" negativa lenta. Se repite 
el segundo estímulo variando las condiciones de registro para hacer patentes los potenciales oscilatorios del brazo ascendente de la onda "b». Se recomienda también un flash de 10 cd.s.m ${ }^{-2}$ y se puede realizar estímulos intermedios con intervalos adecuados. Posteriormente se adapta a la luz (30

\section{ERG-F}

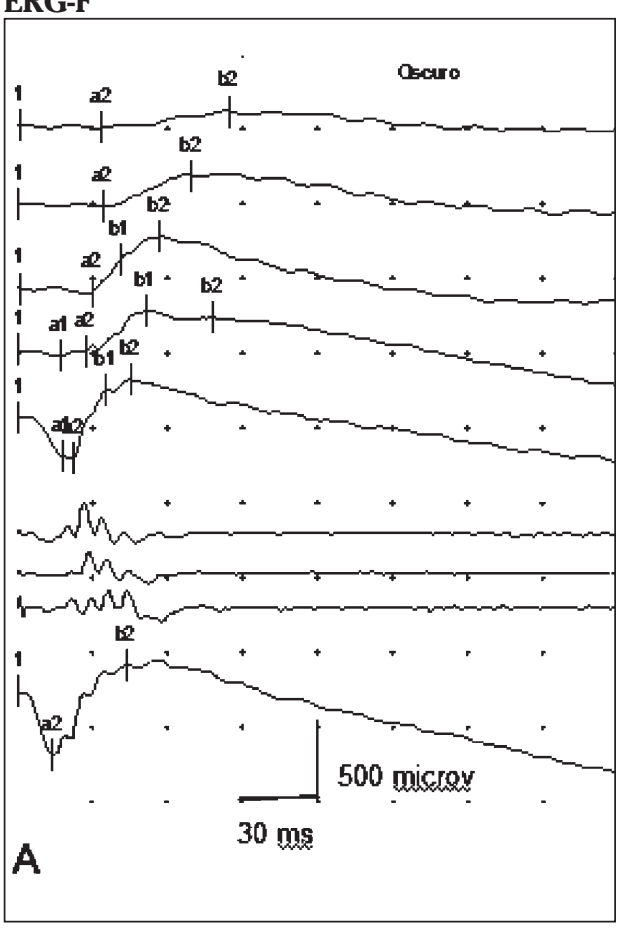

El origen de los componentes ha sido estudiado mediante comparación de resultados de retinas de animales con predominio de conos o de bastones, observando su desaparición con dosis progresivas de éter o de tóxicos cotejando con estudios anatomopatológicos posteriores, con microelectrodos en cultivos celulares y observando las respuestas con distintas condiciones. De todo ello se concluye que la onda "a" depende fundamentalmente de la actividad de los fotorreceptores, la "b» de las células bipolares con contribución de las de Müller, la "c" del epitelio pigmentario, y los potenciales oscilatorios del estrato plexi- cd. $\mathrm{m}^{-2}$ ) y se estimula con 3 cd.s. $\mathrm{m}^{-2}$ a $1 \mathrm{~Hz}$ y a alta frecuencia $(30 \mathrm{~Hz})$ obteniendo en ambos casos respuestas mediadas sólo por conos, que disponen de un mecanismo de adaptación a la luz del que carecen los bastones y de una resolución temporal mucho mayor que éstos (Fig. 1).

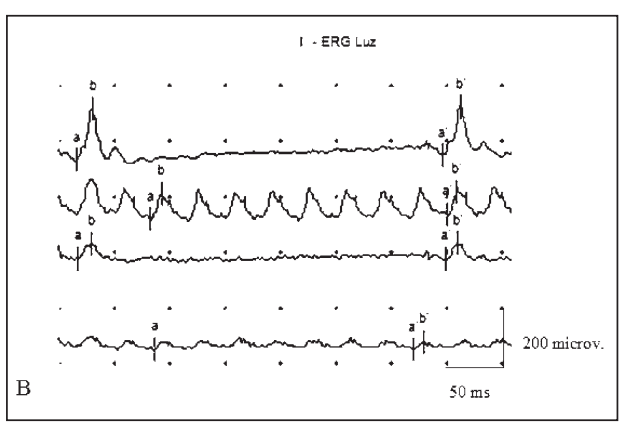

Figura 1. ERG-F. Protocolo ISCEV, ampliado.

A: Ojo adaptado a la oscuridad. Respuestas obtenidas (de arriba a abajo) al estimular con flash de 3 cd.s. $\mathrm{m}^{-2}$ al que se ha añadido los siguientes filtros de color o densidad neutra (dn) medidos en unidades logarítmicas: dn4, dn3, dn1+azul y rojo en las trazas 1 a 4 respectivamente; traza 5 , sin filtro; trazas 6, 7 y 8: potenciales oscilatorios con luz blanca, azul y roja respectivamente. Traza 9: respuesta al estímulo de 10 cd.s.m ${ }^{-2}$.

B: Ojo adaptado a la luz. Respuestas a 3 y $30 \mathrm{~Hz}$ con luz blanca y roja sucesivamente.

forme interno. Las células ganglionares no contribuyen a la respuesta ${ }^{7,10-12}$.

Se puede realizar en niños, pero como la retina no se completa hasta los 6 años, no existen series evolutivas de valores normales y, por otra parte, la mayoría de las veces requiere sedación o anestesia general que también afecta a la amplitud de la respuesta ${ }^{13}$, la valoración es más difícil. Todo ello hace que su indicación en clínica este restringida a casos concretos.

El ERG-F se altera en muchas enfermedades retinianas, sobre todo difusas: distrofias de conos y bastones y de bastones 
y conos tanto en la forma pura como en las sindrómicas, alteraciones estacionarias de los conos, ceguera nocturna estacionaria y sus variantes, distrofias coroido-retinianas $\mathrm{y}$ vítreo-retinianas, déficit de vitamina $\mathrm{A}$, procesos infecciosos, inflamatorios, metabólicos y tóxicos (entre ellos hay que destacar los efectos nocivos de algunos medicamentos como los antipalúdicos de síntesis y algunos antiepilépticos). Sin embargo, en las alteraciones focales, aunque sean de gran importancia funcional como es el caso de las maculopatías, el ERG-F es normal, como también lo es en las alteraciones del NO aunque exista ya una atrofia óptica ${ }^{7,10,11}$.

\section{ELECTRORRETINOGRAMA-PATRÓN}

Electrorretinograma-patrón (ERG-P) es la respuesta obtenida al estimular la retina central con un patrón geométrico, tipo damero o a rayas, reversible, blanco y negro, con características de contraste y luminancia controladas y con un punto de fijación central.

La respuesta consta de un componente positivo a unos $50 \mathrm{~ms}$ (P50) seguido de un negativo a $95 \mathrm{~ms}$ (N95) y precedido de un pequeño negativo a 30-35 ms (N35). (Fig. 2). La amplitud es baja, lo cual hace más difícil su registro. Como para el ERG-F, la ISCEV redactó en 1996 un protocolo que ha ido revisando y cuya última actualización data de $2007^{14}$.

\section{PEV-ERG-P}

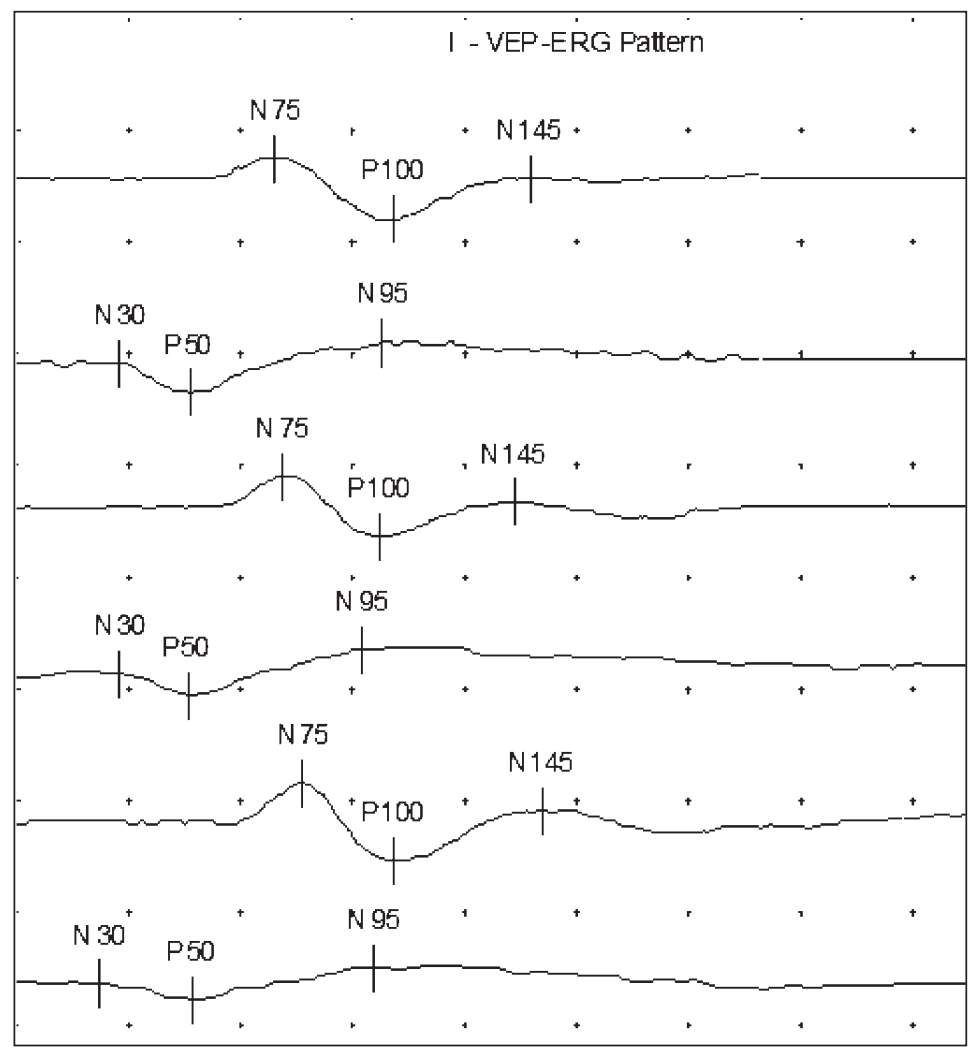

Figura 2. PEV y ERG-P. Registro simultáneo de PEV (trazas impares, derivación Oz-Fpz)) y ERG-P (trazas pares) realizado con patrones de 60, 30 y 15 minutos de ángulo visual. Tiempo de análisis: $300 \mathrm{~ms}$. Amplitud: 50 microV. / división en trazas impares y 20 microV /división en trazas pares. 
Hay varios tipos de electrodos de registro: laminilla de oro "gold foil", de fibra en el borde palpebral "DTL" o para colgar del párpado y hay que colocarlos cuidadosamente de forma que no degraden la imagen visual, motivo por el cual no sirve la lentilla; la referencia en el canto externo del ojo. Los electrodos externos no son útiles. Se estimula 15-18 grados de visión, con cuadrícula de 0,6-1 grado. Hay que realizar el registro con la mejor corrección óptica posible, sin dilatación pupilar para preservar la acomodación, y se precisa una adecuada fijación en el centro. Es necesario promediar al menos 300 estímulos debido a la baja amplitud de la respuesta.

Para determinar el origen del ERG-P, en la década de los 80 se estudió en animales el efecto de la sección del NO: la respuesta ERG-P desaparecía en concordancia con la degeneración de las células ganglionares observada histológicamente mientras la respuesta ERG-F permanecía inalterada; por lo tanto, se originaba en las células ganglionares ${ }^{15}$. Pero en algunas especies animales y en personas sin percepción luminosa por sección traumática del NO lo suficientemente antigua como para haberse producido con seguridad la degeneración ganglionar, se registró una pequeña $\mathrm{P} 50$ con latencia acortada; es decir, no toda la respuesta ERG-F depende de las células ganglionares ${ }^{16}$. Otros estudios demuestran que la respuesta es distinta si la alteración está en la mácula o en el $\mathrm{NO}^{17-}$ ${ }^{19}$, y sugieren distinto generador para P50 y N95. Hoy día se acepta que N95 depende de las células ganglionares y tiene relación con el contraste y P50 depende de estratos más externos.

El descenso de amplitud de P50 indica disfunción de la mácula previa a las células ganglionares; en casos severos, con alteración visible en el fondo de ojo, llega a la extinción. Puede ir acompañado de reducción de N95, en menor grado. Si se acompaña de descenso de las respuestas de conos del ERG-F, no es una maculopatía pura sino una disfunción generalizada de conos; si también están alteradas las respuestas en oscuridad, es disfunción de conos y bastones. El descenso de P50 con descenso de onda "a" sugiere alteración de fotorreceptores; con un ERG-F negativo indica alteración postfototransducción ${ }^{18,19}$. Si el descenso es de N95 indica afectación de las células ganglionares y se acompaña de alteración del PEV (es el caso de las neuropatías ópticas, si bien puede existir neuropatía con ERG-P poco alterado o normal).

Las patologías más frecuentes de degeneración primaria de las células ganglionares son la atrofia óptica hereditaria de Leber (LHON), debida a una mutación en el genoma mitocondrial, y la atrofia óptica dominante tipo Kjer (DOA, Kjer), por alteración del gen OPA1. En ambas hay descenso de la amplitud del PEV, del componente N 95 del ERG-P y en fases avanzadas también del P50, con descenso de amplitud y de latencia pero sin llegar a desaparecer ${ }^{19}$.

El ERG-P ayuda al diagnóstico y seguimiento del glaucoma ${ }^{20}$, sobre todo cuando se utiliza estimulando a frecuencias altas: $16 \mathrm{~Hz}$. Ventura y Porciatti ${ }^{21}$ han diseñado un paradigma, PERGLA, que aporta la comodidad de poder registrar la señal con electrodos externos, pero requiere un estimulador más sofisticado y un programa que incluya la transformada de Fourier, posibilidad que no esta al alcance de todos los equipos.

\section{ELECTRORRETINOGRAMA- MULTIFOCAL}

El electrorretinograma-multifocal (ERGMF) permite registrar la respuesta global de los conos dividida topográficamente en respuestas focales, en condiciones de adaptación a la luz. El estimulo consiste en 61 ó 103 hexágonos de distintos tamaños según la zona de la retina que van a estimular, calculados para producir respuestas de amplitud similar y se activan en una secuencia pseudoaleatoria. El programa, mediante un algoritmo matemático complejo, extrae la respuesta asociada a cada uno de los hexágonos. Hay que tener en cuenta que no son potenciales directos obtenidos de cada zona de la retina sino un cálculo matemático. 
El método de registro es similar al ERGF de luz, pero precisa del estimulador y analizador correspondientes, y de una adecuada fijación por parte del paciente. Es una técnica en auge, útil para el estudio de la retina central pero todavía no de uso común ${ }^{22}$.

La ISCEV ha publicado y revisado una guía pero no es un estándar porque aún está en evolución ${ }^{23}$.

\section{ELECTROOCULOGRAMA}

El epitelio pigmentario de la retina (EPR) es una capa de células, situada por fuera de los fotorreceptores, que contribuye a mantener la retina externa. Entre sus funciones está absorber la luz sobrante para evitar que los fotorreceptores se reestimulen, transportarles el retinol en el ciclo de los fotopigmentos, fagocitar los discos que se desprenden del segmento externo de los fotorreceptores, transportar fluidos y metabolitos, regular el contenido del cytosol y, también, forma parte de la barrera hemato-retiniana externa.

El EPR es responsable de la diferencia de potencial existente entre la córnea y el fondo del ojo, formando un dipolo en el eje óptico, positivo en la córnea y negativo en el fondo. Este potencial se puede registrar mediante electrodos colocados en la piel cerca del ojo ya que varía según el ángulo de rotación del ojo, hecho que se utiliza para estudiar la motilidad ocular. Además, el potencial córneo-fundal del ojo a oscuras se modifica considerablemente con la luz. El registro del potencial y su comportamiento en oscuridad y con luz es la base del electrooculograma ${ }^{24,25}$.

También para el EOG hay un protocolo estándar de la ISCEV ${ }^{26}$. El registro se realiza con electrodos colocados en los cantos de los ojos, con las pupilas dilatadas, apoyando la barbilla en un estimulador "ganzfeld" que posee dos puntos de luz, que se encienden alternativamente, cada uno a 15 minutos angulares del punto central. Con la cabeza quieta, se mira la luz; al mover el ojo cambia la dirección del vector eléctrico, hecho que es captado por los electro- dos en forma de una onda cuadrada. Después de haber estado con luz ambiental media, se realiza el registro en oscuridad durante 15 minutos y luego otros 15 minutos con luz; los puntos de luz alternantes aparecen durante 15 segundos cada minuto y se mide la amplitud de las respuestas de cada serie (Fig. 3). Lógicamente, en las personas con nistagmus u otra alteración de la motilidad ocular, escotoma central, fotofobia o incapacidad para mantener la atención, no se puede realizar adecuadamente esta medición.

Existe un potencial basal que en la oscuridad decrece hasta alcanzar un mínimo. Si a continuación se ilumina la retina, tras un descenso inicial hay una elevación durante 7-14 minutos (respuesta a la luz) para luego descender más lentamente. La relación entre el máximo alcanzado en luz y el mínimo en la oscuridad se denomina Índice de Arden. No se conoce bien el mecanismo que da lugar al pico con luz, pero sí que hay una serie de pasos: se inicia en los fotorreceptores, cambia la concentración de una sustancia no identificada a nivel subretiniano, se activa un receptor de la membrana apical, se desencadena una cascada intracelular que se modifica por Best1 y se activa el canal de cloro de la membrana basal, despolarizándola ${ }^{25}$. La respuesta normal requiere la normalidad de los fotorreceptores, del EPR y de los mecanismos entre ambos.

La ingesta de alcohol produce una respuesta similar, con un retraso de unos tres minutos, que es el tiempo que tarda en llegar a la sangre. La adaptación a la luz continua no inhibe la respuesta del alcohol, sí la de la luz; la persistencia de un nivel de alcohol en sangre no inhibe la de la luz, sí la del alcohol. Dosis submáximas de ambos estímulos a la vez hacen que las respuestas se sumen, pero dosis altas producen oclusión de la respuesta. Por tanto, deben tener mecanismos distintos en su inicio y convergen en uno común ${ }^{27}$. No obstante, las respuestas obtenidas con luz, y más aún con alcohol, son muy variables entre unos individuos y otros y también dentro del mismo individuo, lo cual resta utilidad clínica ${ }^{28}$. 


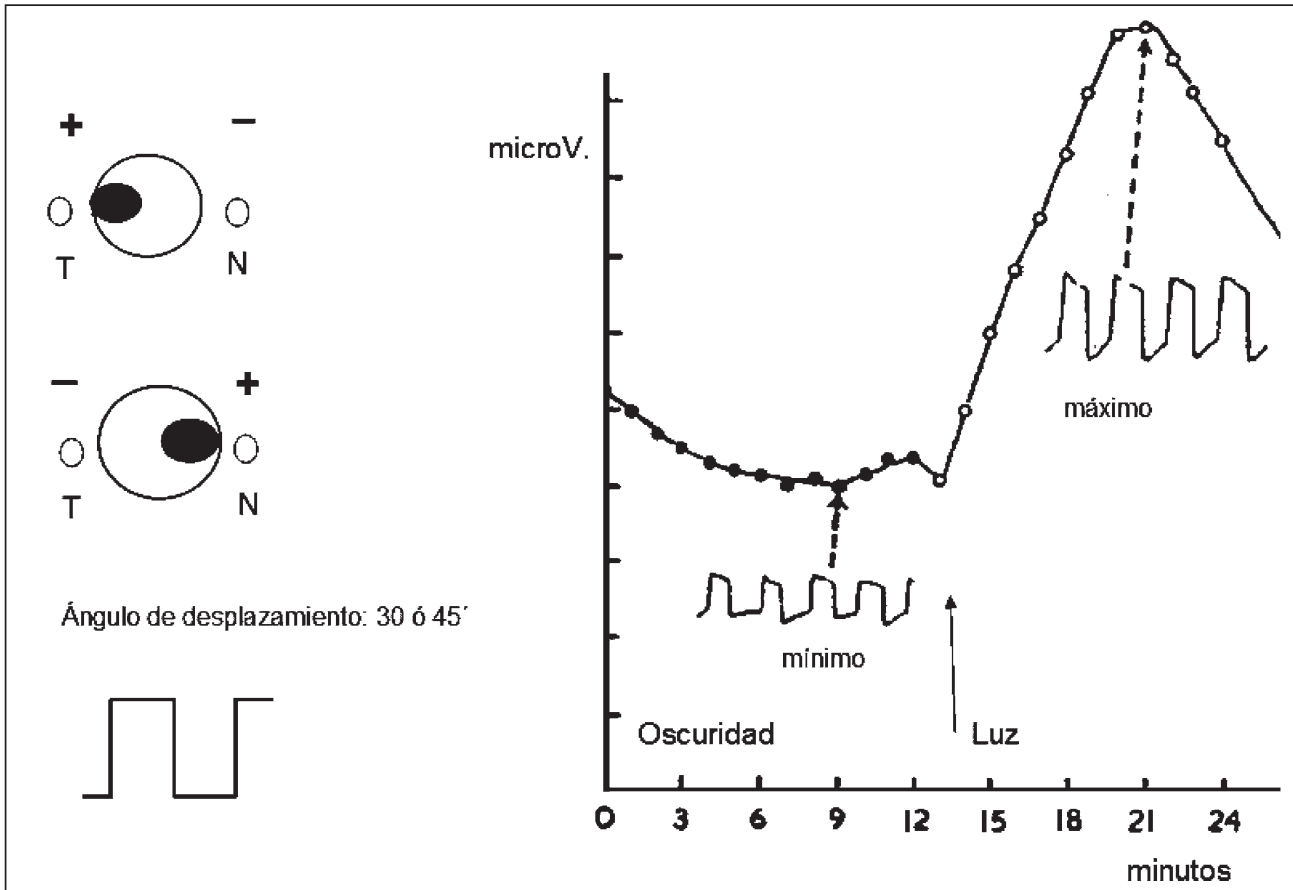

Índice de Arden: amplitud máxima en luz / amplitud mínima en oscuridad.

Figura 3. EOG. A la izquierda representación esquemática del ojo derecho con electrodo nasal (N) y temporal (T). Al desplazar el ojo hacia la derecha, T recoge la positividad de la córnea respecto a la negatividad fundal recogida por $\mathrm{N}$; al desplazarlo hacia la izquierda ocurre lo contrario y se registra una onda "cuadrada". A la derecha vemos la amplitud de cada serie a lo largo del tiempo, primero durante 13 minutos en oscuridad y luego con luz.

El EOG es dato de valor diagnóstico en la distrofia macular viteliforme de Best; en otras alteraciones maculares o del EPR está dentro de valores normales o no concluyentes $^{28,29}$. En patologías difusas como la retinosis pigmentaria se altera, pero es mucho menos sensible que el ERG-F.

La enfermedad de Best se ha diagnosticado clásicamente por las alteraciones del fondo de ojo, herencia autosómica dominante, evolución clínica variable, ERG-F normal, y la presencia, incluso en estadío preclínico, de un EOG patológico con índice de Arden, menor de 1,5. Actualmente se sabe que es una mutación en el gen Best1, localizado en el cromosoma 11 (11q13).
También la vitreocoroidopatía autosómica dominante, la bestrofinopatía autosómica recesiva y la degeneración macular viteliforme del adulto se deben a mutaciones del gen Best1 pero cursan con EOG normal. Algunos datos apuntan a una alteración de los canales de cloro de la membrana basal del EPR, que influyen en el calcio, pero no son ni mucho menos concluyentes ${ }^{29}$.

\section{POTENCIALES EVOCADOS VISUALES}

Potencial evocado visual (PEV) es la respuesta producida en la corteza cerebral por los estímulos visuales. Para poder estudiarla se aplican estímulos breves, de 
parámetros controlados y se registra con electrodos colocados en el cuero cabelludo. Como está inmersa en la actividad electroencefalográfica, se necesita técnicas de premediación para hacerla visible. La respuesta obtenida esta originada por las fibras que provienen de la retina central; por tanto, depende de la integridad del ojo, retina, NO, quiasma, radiaciones ópticas y córtex occipital.

La forma de estimulación más habitual es mediante patrón reversible, el mismo que se utiliza para ERG-P y se realizan series con cuadriculas de distintos tamaños, es decir, con varios ángulos visuales. Se precisa una correcta fijación y la mejor corrección óptica posible. En caso de no conseguir una fijación adecuada o en niños pequeños se utiliza el flash, pero su valor es mucho menor. Se estimulan ambos ojos, por separado. Tanto la Sociedad Americana de Neurofisiología Clínica como la ISCEV han publicados y revisado protocolos, siendo los más recientes de $2006^{30}$ y $2009^{31}$ respectivamente.

Las respuestas se recogen mediante electrodos colocados en la región occipital con referencia a un electrodo frontal medio. Dada la organización retinotópica de la corteza visual primaria, el electrodo occipital medio capta la respuesta que el estímulo de la zona foveal evoca en la corteza estriada y consta de una prominente onda positiva con una latencia media de $100 \mathrm{~ms}$, precedida y seguida de componentes negativos a 75 y 145 ms (N75-P100-N145) (Fig. 2). El mismo electrodo medio sirve para las respuestas de uno y otro ojo, facilitando la comparación entre ellas ${ }^{5,30}$. Por la misma razón los electrodos laterales son más útiles cuando se realiza estimulación por hemicampos. Pero se da una paradoja: como el estímulo de un hemicampo llega al lóbulo occipital contralateral sería de esperar una P100 contralateral y, sin embargo, se obtiene ipsilateral, debido a la orientación oblicua de la corteza occipital; la P contralateral aparece a $135 \mathrm{~ms}^{30}$.

La mayor utilidad del PEV es el estudio de la patología del NO ya que en caso de afectación unilateral las respuestas me- diadas por el ojo sano serán normales y las mediadas por el afectado estarán alteradas. Cuando existe desmielinización en el NO, el PEV aparece retrasado y, con frecuencia, no se acompaña de descenso de amplitud de la respuesta. El retraso persiste incluso después de la recuperación de los síntomas y puede poner de manifiesto alteraciones subclínicas ${ }^{32}$. Sin embargo, en la neuropatía óptica isquémica se observa un descenso de la amplitud de la respuesta con menor o nula alteración de la latencia.

Si la afectación es de ambos NO, quiasmática o postquiasmática, se alteran las respuestas de uno y otro ojo perdiendo el valor localizador. En estos casos la estimulación por hemicampos ayuda al diagnóstico topográfico ${ }^{30,33-35}$.

El registro simultáneo del PEV y ERG-P es muy útil para diferenciar si la alteración es macular o del $\mathrm{NO}^{5,33-36}$.

Hay que tener en cuenta que todas estas técnicas precisan que la luz llegue a la retina adecuadamente $\mathrm{y}$, por tanto, pueden alterarse en patología ocular previa a la retina.

\section{BIBLIOGRAFÍA}

1. Tessier-Lavigne M. El procesamiento visual en la retina. En: Kandel ER, Schwartz JH, Jessell TM editores. Principios de neurociencia (4 $4^{\mathrm{a}}$ ed). McGraw-Hill/Interamericana 2000; 507522 .

2. Sharma JK, Ehinger BEJ. Development and structure of the retina. En: Kaufman PL, Alm A, editores. Adler's physiology of the eye (10 ed). St Louis: Mosby Inc 2003; 319-347.

3. BERSON EL. Visual function testing: clinical correlations. J Clin Neurophysiol 1994; 11: 472481.

4. Celesia GG, DeMarco PJ Jr. Anatomy and physiology of the visual system. J Clin Neurophysiol 1994; 11:482-492.

5. Tobimatsu S, Celesia GG. Studies of human pathophysiology with visual evoked potentials. Clin Neurophysiol 2006; 117: 1414-1433.

6. Wurtz RH, KANDEL ER. Vias visuales centrales. En: Kandel ER, Schwartz JH, Jessell TM editores. Principios de neurociencia ( $4^{\mathrm{a}}$ ed). McGraw-Hill/Interamericana. 2000; 523-547. 
7. Ponjavic V, AndrÉAsson S. Electrophysiology and retinal function. En: Kaufman PL, Alm A, editores. Adler's physiology of the eye $\left(10^{\mathrm{a}}\right.$ ed). St Louis Mosby 2003: 409-421.

8. Marmor MF, Fulton AB, Holder Ge, Miyake Y, BRIGELL M, BACH M (for the International Society for Clinical Electrophysiology of Vision). ISCEV Standard for full-field electroretinography (2008 update). Doc Ophthalmol 2009; 118: 69-77.

9. Papathanasiou ES, Papacostas SS. Flash electroretinography: normative values with surface skin electrodes and no pupil dilatation using a standard stimulation protocol. Doc Ophthalmol 2008; 116: 61-73.

10. Fishman GA. The electroretinogram. En: Fishman GA, Birch DG, Holder GE, Brigell MG. Electrophysiologic testing in disorders of the retina, optic nerve and visual pathway. ( $2^{\mathrm{a}}$ ed). Ophthalmology Monograph 2. The Foundation of the American Academy of Ophthalmology, San Francisco 2001: 1-155.

11. Armington JC. Electroretinography. En: Aminoff MJ editor. Electrodiagnosis in clinical neurology ( $3^{\mathrm{a}}$ ed) Churchill Livingston. 1992; 433-466.

12. Robson JG, Frishman LJ. Dissecting the darkadapted electroretinogram. Doc Ophthalmol 1999; 95: 187-215.

13. Tremblay F, Parkinson JE. Alteracion of electroretinografic recordings when performed under sedation or halogenate anesthesia in a pediatric population. Doc Ophthalmol 2003; 107: 271-279.

14. Holder GE, Brigell MG, Hawlina M, Meigen T, VAEGAN, BACH M. (for the International Society for Clinical Electrophysiology of Vision). ISCEV Standard for clinical pattern electroretinography-2007 update. Doc Ophyhalmol 2007; 114: 111-116.

15. Maffei L, Fiorentini A, Bisti S, Hollander H. Pattern ERG in the monkey after section of the optic nerve. Exp Brain Res 1985; 59; 423-425.

16. HaRrison JM, O`Connor PS, Young RSL, Kincaid M, Bentley R. The pattern ERG in man following surgical resection of the optic nerve. Invest Ophthalmol Vis Sci 1987; 28: 492-499.

17. Ryan S, Arden GB. Electrophysiological discrimination between retinal and optic nerve disorders. Doc Ophthalmol 1988; 68: 247-255.

18. HoLDER GE. The pattern electroretinography. En: Fishman GA, Birch DG, Holder GE, Brigell MG. Electrophysiologic testing in disorders of the retina, optic nerve and visual pathway ( $2^{\mathrm{a}}$ ed). Ophthalmology Monograph 2. The Foundation of the American Academy of Ophthalmology, San Francisco 2001: 197-235.

19. Holder GE. Pattern electroretinography (PERG) and an integrated approach to visual pathway diagnosis. Prog Ret Eye Res 2001; 20: 531-561.

20. Bach M, Hoffmann MB. Update on the pattern electroretinogram in glaucoma. Optom Vis Sci 2008; 85: 386-395.

21. Porciatti V, Ventura LM. Normative data for a user-friendly paradigm for pattern electroretinogram recording. Ophthalmology 2004; 111: 161-168.

22. Hood DC, Odel JG, Ghen CS, Winn BJ. The multifocal electroretinogram. J Neuro-Ophthalmol 2003; 23: 225-235.

23. Hood DC, Bach M, Brigell M, Keating D, Kondo $\mathrm{M}$, LYoNs JS et al. ISCEV guidelines for clinical multifocal electroretinography (2007 edition). Doc Ophthalmol 2008; 116: 1-11.

24. Arden Gb, Kelsey JH. Changes produced by light in the standing potential of the human eye. J Physiol 1962; 161: 189-204.

25. Arden GB, Constable PA. The electr-oculogram. Prog Ret Eye Res 2006; 25: 207-248.

26. Brown M, Marmor M, VAegan, ZREnNer E, Brigell M, BACH M. ISCEV standard for clinical electro-oculography (EOG) 2006. Doc Ophthalmol 2006; 113: 205-212.

27. Wolf JE, Arden B. Two components of the human alcohol electro-oculogram. Doc Ophthalmol 2004; 109: 123-130.

28. Marmor MF, Wu HC. Alcohol and light-induced electro-oculographic responses: variability and clinical utility. Doc Ophthalmol 2005; 110: 227-236.

29. Marmorstein AD, Cross HE, Peachey NS. Functional roles of bestrophins in ocular epithelia. Prog Ret Eye Res 2009; 28: 206-226.

30. EPSTEIN CM et al. Recommended standards for visual evoked potentials. American clinical neurophysiology society. Guideline 9B: guidelines on visual evoked potentials. J Clin Neurophysiol 2006; 23: 138-156.

31. Odom JV, Bach M, Brigell M, Holder GG, McCuLLOCH DL TORMENE AP et al. ISCEV standard for clinical visual evoked potentials (2009 update). VEP standard 2009v81.rtf

32. Halliday AM, McDonald WI, Mushin J. Visual evoked responses in the diagnosis of multiple sclerosis. Br Med J 1973; 4: 661-664. 
33. ChIAPPA KH, HiLL RA. Pattern-shift visual evoked potentials. Interpretation En Chiappa $\mathrm{KH}$ editor. Evoked potentials in clinical Medicine ( $3^{\text {a }}$ ed). Lippincott-Raven 1997; 95-130.

34. BRIGELL MG. The visual evoked potential. En: Fishman GA, Birch DG, Holder GE, Brigell MG. Electrophysiologic testing in disorders of the retina, optic nerve and visual pathway ( $2^{\mathrm{a}}$ ed). Ophthalmology Monograph 2. The foundation of the american academy of ophthalmology, San Francisco 2001: 237-278.

35. Walsh P, Kane N, Butler S. The clinical role of evoked potentials. J Neurol Neurosurg Psychiatry 2005; 76: 16-22.

36. Holder GE, Gale RP, Acheson JF, Robson AG. Electrodiagnostic assessment in optic nerve disease. Curr Opin Neurol 2009; 22: 3-10. 